\title{
The box alignment illusion: An orientation illusion induced by pictorial depth
}

\author{
JAMES T. ENNS and STANLEY COREN \\ University of British Columbia, Vancouver, British Columbia, Canada
}

\begin{abstract}
In four experiments, observers attempted to align two sets of oblique edges to parallel. The contexts for these alignments included lines in isolation (2-D control), lines embedded in orthogonal drawings of same-oriented and different-oriented boxes (3-D objects), and each of these viewed against backgrounds depicting strong linear perspective (3-D backgrounds). A consistent distortion was observed in the alignments of different-oriented boxes relative to control lines, indicating that the parallel lines in these stimuli appeared to diverge toward the top of the picture. Furthermore, this box alignment illusion decreased with interstimulus distance, whereas alignment distortions in control lines and sameoriented boxes increased with distance. Viewing the stimuli against 3-D backgrounds produced a dramatic reversal of the illusion, with control lines now appearing to converge more than the boxes. These results suggest that the illusion reflects basic processes involved in pictorial depth perception.
\end{abstract}

Line drawings are impoverished and ambiguous sources of information. They are simpler than the corresponding view of the three-dimensional (3-D) array in many ways, including their elimination of extraneous contours found in real images and their lack of some real-world depth cues (e.g., binocular disparity, motion parallax). They are ambiguous in that to perceive the depicted depth, one must isolate and emphasize some cues (e.g., linear perspective, interposition, and shadowing) while ignoring or suppressing others (e.g., accommodation, binocular disparity, convergence, motion parallax, and surface texture). Simply put, the observer must opt for the 3-D interpretation implied by certain markings on the pictorial surface rather than the percept of the flat surface itself.

Despite these apparent complexities, humans have a remarkable ability to rapidly and accurately interpret depicted shape and depth in line drawings (e.g., Biederman, 1987; Butler \& Kring, 1987; Deregowski, 1989; Enns \& Rensink, 1990a, 1990b, 1991; Kennedy, 1974; Perkins, 1972; Wade, 1990; Weisstein \& Harris, 1974). One of the more striking demonstrations of this ability can be seen in a visual search task in which the target and distracter items are the drawings of 3-D boxes shown in Figures $1 \mathrm{C}$ and $1 \mathrm{~F}$ (Enns \& Rensink, 1990b). While response times varied little as a function of the number of these boxes present (i.e., less than $10 \mathrm{msec}$ per item), they increased markedly (i.e., more than $30 \mathrm{msec}$ per item) in a similar task involving items lacking in 3-D appearance (e.g., Figure 1D).

This research was supported by grants from the Natural Sciences and Engineering Research Council of Canada to both authors. We kindly acknowledge Kevin Medland, Diana Ellis, Carmen Stoessel, and Tracey Wood, who assisted in data collection. For reprints or other correspondence, contact J. T. Enns, Department of Psychology, University of British Columbia, 2136 West Mall, Vancouver, B.C., Canada V6T $1 Z 4$ (e-mail: jenns@cortex.psych.ubc.ca).
Data such as these demonstrate that some aspects of 3-D perception are carried out rapidly and automatically, yet, they do not indicate how the visual system performs this feat. There is some consensus, however, that several mechanisms are involved. One involves the isolation of specific cues, both implicit and explicit, which have a degree of ecological validity when viewing 3 -D scenes and which can also be approximated in 2-D arrays (Deregowski, 1989; Gibson, 1979; Kennedy, 1974; Niall \& Macnamara, 1990). Other mechanisms must invoke certain assumptions concerning the 3-D world to help interpret these cues, such as that scenes are generally lit from above (Kleffner \& Ramachandran, 1992; Ramachandran, 1988), that trihedral junctions in a picture normally indicate 3-D corners (Enns \& Rensink, 1991; Perkins, 1972), that objects are generally convex rather than concave (Gregory, 1970; Howard, 1982), and that height in the picture plane is associated with relative distance (Bruno \& Cutting, 1988). Some of these assumptions may reflect the legacy of species evolution, while others are almost certainly the product of experience in specific environments. In any case, once the cues have been isolated and the various processing assumptions applied to produce the perception of depth, the resultant information can be fed into constancy scaling mechanisms to provide some sense of the size, shape, distance, and brightness of objects in the actual scene (see Coren, 1990).

Reliance upon specific depth cues and processing strategies can be inappropriate in certain contexts, thereby causing systematic distortions known as the visual-geometric illusions (Coren, 1991; Coren \& Girgus, 1978). For example, inappropriate constancy scaling has been used to explain a number of illusions, including the Müller-Lyer (e.g., Gregory, 1970; Warren \& Bashford, 1977), Ponzo (e.g., Coren \& Girgus, 1977), Poggendorff (e.g., Gillam, 1971, 1980; Parks \& Hui, 1989), horizontal-vertical (e.g., Girgus \& Coren, 1975; von Collani, 1985), subjective contours (e.g., Coren, 1972; Coren \& Porac, 1983b; Purghé \& 


\section{Stimulus pairs with parallel oblique lines}

\section{Parallel edges thatt appear to diverge}
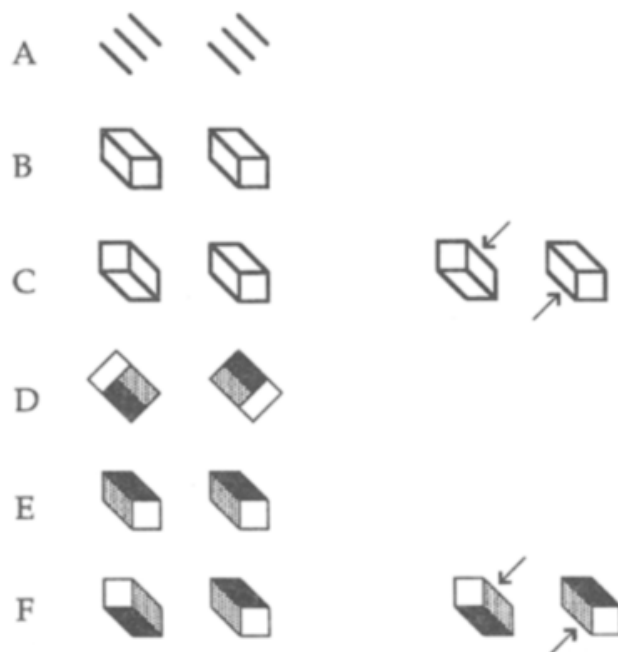

Figure 1. The six stimulus pairs used in Experiment 1. Pairs $C$ and $F$ have previously served as items in visual search tasks demonstrating "pop-out" (Enns \& Rensink, 1990b). Arrows point to two physically parallel edges in $C$ and $F$ that appear to diverge toward the top of the page.

Coren, 1992), the moon illusion (e.g., Coren, 1992; Coren \& Aks, 1990; Kaufman \& Rock, 1989; Reed, 1984), and others (e.g., Borresen, 1990; Coren \& Girgus, 1975, 1978; Ward, Porac, Coren, \& Girgus, 1977).

In some instances, the strength of an illusion can be directly related to the number and saliency of depth cues (e.g.,

Coren \& Aks, 1990). Figure 2 shows an example of this principle for the Müller-Lyer illusion, where the presence of depth cues causes a much greater apparent length difference in the two heavy vertical lines than is usually observed in the classical form of the illusion (after Coren, Ward, \& Enns, 1994). In other situations, the manipulation of pictorial depth cues can be used to attenuate or even reverse the direction of the illusion (e.g., Coren, 1992; Girgus \& Coren, 1973; Gregory \& Harris, 1974; Lawson, Cowan, Gibbs, \& Whitmore, 1974; Vurpillot, 1963).

The notion that some depth cues are processed involuntarily has been used most successfully to explain illusions of relative size (e.g., Müller-Lyer, Ponzo, moon), subjective contours (e.g., Kanisza figures), and displacement (e.g., Poggendorff). Illusions involving distortions of relative orientation have seldom been dealt with by invoking depth processing (e.g., Carpenter \& Blakemore, 1973; Coren \& Hoy, 1986; Rochlin, 1955; but see also Hotopf, Hibberd, \& Brown, 1983, and Wenderoth \& Johnson, 1985). However, it is easy to predict certain orientation illusions based upon such cues. For example, in Figure 3, the upper intersection of the vertical line is actually a right angle although it appears to be oblique, whereas the lower intersection is actually oblique and yet appears to be a right angle. This is undoubtedly related to the presence of pictorial depth cues in the line drawing.

We informally noticed an illusion of line orientation that has apparently not been described elsewhere. It is of interest because it too seems to be related to the involuntary processing of depth cues. To see this illusion, we must turn back to the items depicted as Figures IC and IF. Each figure appears to contain a pair of boxes, with cues suggesting that the specific orientation in space is different for the

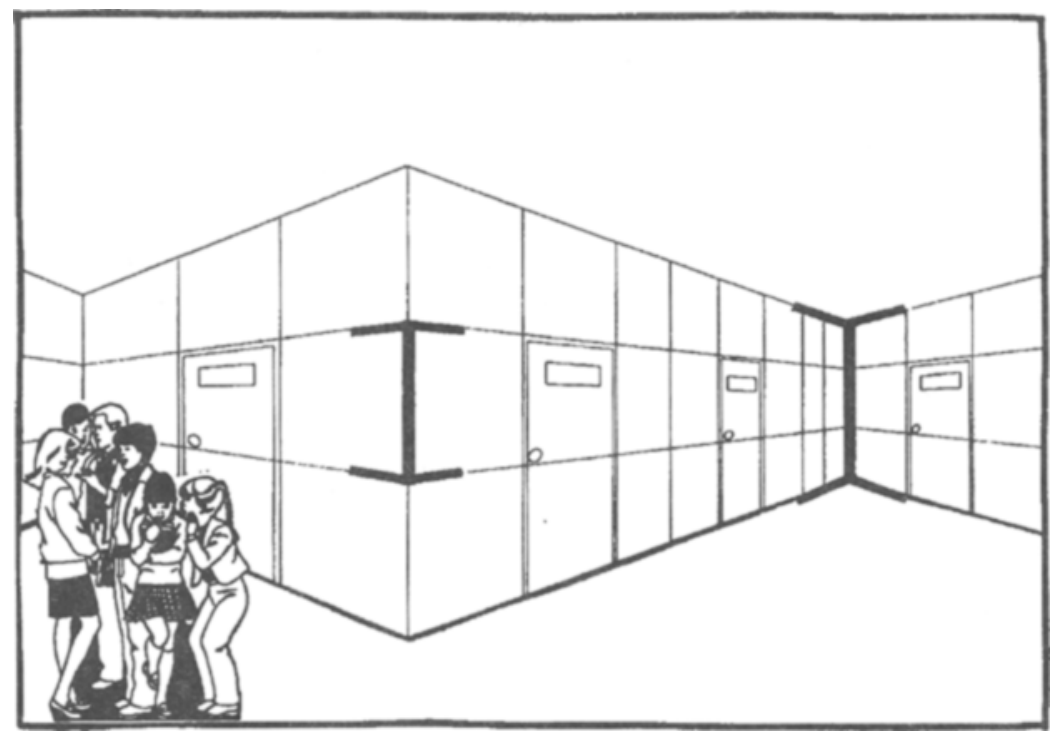

Figure 2. Enhancing depth cues causes an increase in the magnitude of the MüllerLyer illusion. Here, the heavy vertical line with wings out (in the far corner) is the same length as the heavy vertical line with wings in (on the near edge) although it looks to be much longer. Figure from Sensation and Perception (4th ed.), by Stanley Coren, Lawrence M. Ward, and James T. Enns, copyright 1994 by Harcourt Brace \& Co., reproduced by permission of the publisher. 


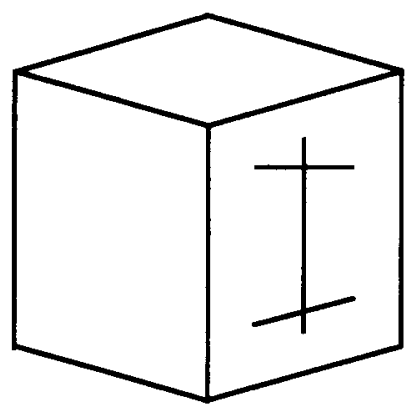

Figure 3. Because of the depth cues implied in this drawing, the upper intersection of the vertical line, which is actually a right angle, appears to be oblique, whereas the lower intersection, which is oblique, appears to be a right angle.

two objects. Now, shift your attention to the oblique lines describing the edges of each box that are nearest to one another (i.e., the inside edges indicated with arrows in Figures $1 \mathrm{C}$ and $\mathrm{IF}$ ). These edges do not appear to be parallel, despite the fact that they have been drawn with physically parallel lines. Rather, the extensions of these edges appear to diverge as one moves toward the top of the picture. To help convince yourself that this is an illusion, turn the page upside down. The designated edges still appear to diverge toward the top of the page, even though they have been rotated by $180^{\circ}$ and should now converge if they were not parallel. We will label this distortion of apparent orientation the box alignment illusion.

The present series of studies began with an attempt to formally establish the existence and strength of the box alignment illusion, as demonstrated in Figures $1 \mathrm{C}$ and $1 \mathrm{~F}$, through the use of controlled tests (Experiment 1). Once the illusion was shown to be reliably obtained, the second goal was to explore some of the factors affecting it. How important is the depicted 3-D orientation difference between the boxes in determining the strength of the illusion? Is the illusion affected by the spatial separation between the two stimuli being compared? These questions were examined in Experiment 2. Finally, we asked whether the box alignment illusion was also affected by the pres- ence of pictorial depth in the surrounding visual field. This question and some of its ramifications were addressed in Experiments 3 and 4.

\section{EXPERIMENT 1 Measurement of the Box Alignment Illusion}

A method of adjustment was used to measure the apparent orientation of the oblique edges in order to demonstrate the existence of the box alignment illusion.

\section{Method}

Stimuli and Apparatus. The stimuli tested are shown in Figures $1 \mathrm{~A}-1 \mathrm{~F}$. The oblique parallel lines in Figure $1 \mathrm{~A}$ (control lines) and the oblique rectangles in Figure $1 \mathrm{D}$ (control shading) were used to establish a baseline of accuracy against which the other stimulus pairs could be compared (Figure 1B, same-oriented line boxes; Figure $1 \mathrm{C}$, different-oriented line boxes; Figure 1E, same-oriented shaded boxes; Figure 1F, different-oriented shaded boxes). In the method of adjustment used here, observers are expected to null their illusions in their settings. This means that if they experienced the same illusion that we observed under informal viewing, then their attempts to align the different-oriented boxes should actually result in the extensions of the lines converging toward the top of the display (thus compensating for or nulling the illusory divergence).

Pairs of test stimuli were centered on $11-\mathrm{cm}$ transparent Plexiglas disks, which in turn were placed side-by-side on a larger $32.5-\mathrm{cm}$ opaque background disk. An example of a stimulus pair is shown in Figure 4 . The center-to-center distance between test stimuli was $16 \mathrm{~cm}$, and the test lines themselves were $1.2 \mathrm{~cm}$ in length. The disk on the right was fixed, such that the oblique lines placed on it had a negative slope of $45^{\circ}$ from vertical. The disk on the left was mounted on a pin that allowed it to be rotated freely. The pin was fixed to an indicator on the rear of the apparatus, allowing angular orientation to be read directly from a fixed protractor. The scale was set so that $0^{\circ}$ corresponded to a setting of parallel. A positive deviation meant that the observer set the oblique lines to converge, thereby indicating compensation (nulling) for an illusion of divergence in the stimulus (as in Figures $1 \mathrm{C}$ and $\mathrm{IE}$ ). Similarly, a negative setting reflected a divergent alignment, corresponding to an illusion of convergence.

Subjects. Twenty-one undergraduates ( 13 females, 8 males) from the university subject pool participated in return for partial course credit in psychology. All reported normal or corrected-to-normal acuity, and all were naive as to the purpose of the experiment.

Procedure. Observers made five settings for each of the six stimulus pairs in a random order. At the beginning of each trial, the ro-

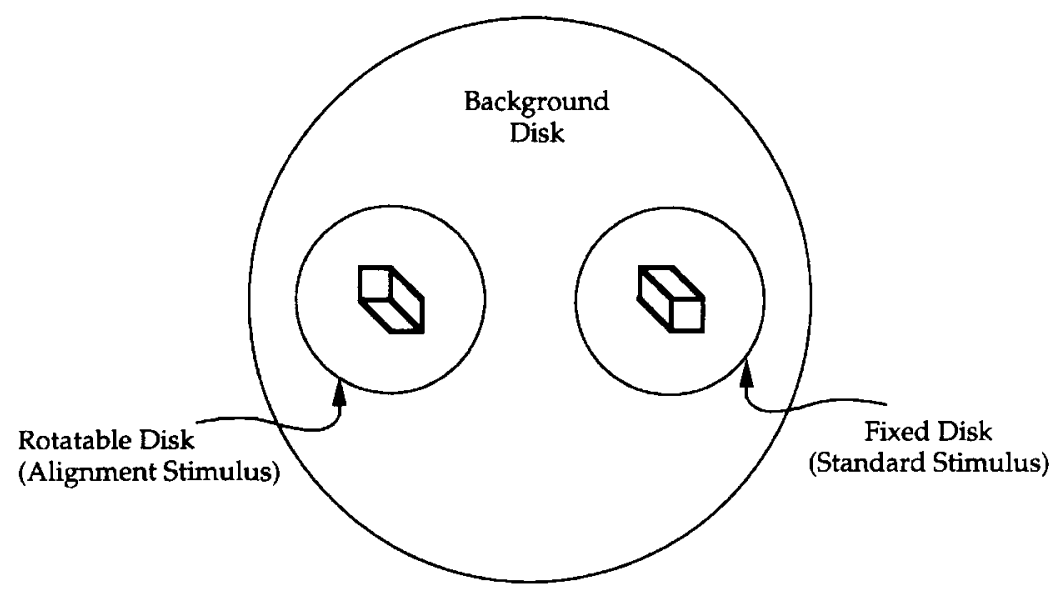

Figure 4. A schematic of the apparatus used in the alignment task in this study. 
tatable disk was placed at either an extreme converging position or an extreme diverging position in an alternating fashion. The large background disk was oriented so that it was perpendicular to the observer's line of sight. The experimenter was blind to the hypotheses under investigation.

The observers were instructed that the stimulus on each side of the display would always contain three parallel lines. These three lines were pointed out by the experimenter for each of the stimulus sets prior to testing. The observers were then shown how to rotate the moveable disk and told that they would be asked to realign the disk on each trial so that the sets of lines on each side of the display were as close to parallel as possible. The experimenter kept the values recorded on each trial hidden from the observer's view.

\section{Results and Discussion}

The mean alignment scores are shown in Table 1, with the values indicating the degree of distortion from parallel (0 degrees). An analysis of variance (ANOVA) revealed significant differences among the stimuli $[F(2,40)=17.04$, $\left.M S_{\mathrm{e}}=4.588, p<.001\right]$, but not between the line-drawn versus shaded drawings overall $\left[F(1,20)=1.34, M S_{\mathrm{e}}=\right.$ 5.921]. The stimulus $\times$ drawing type interaction was marginally significant $\left[F(2,40)=2.77, M S_{\mathrm{e}}=4.950, p<.07\right]$ and its nature became obvious when we computed the mean illusion magnitude for each box stimulus. This was done by subtracting the settings for the control lines (Figure 1A) from the same-orientation (Figure 1B) and differentorientation (Figure 1C) outline boxes and by subtracting the settings for the shading control (Figure 1D) from the sameorientation (Figure 1E) and different-orientation (Figure 1F) shaded boxes. The resulting values are shown in Figure 5, where a positive score (above the axis) indicates the expected illusory divergence toward the top of the picture.

Planned comparisons revealed a significant illusion for both the same-oriented line boxes $[t(20)=4.14, p<.01]$ and the different-oriented line boxes $[t(20)=4.34, p<$ .011 . The difference between these conditions was not reliable [mean difference $=0.14^{\circ}, t(20)=0.51$ ]. However, a different pattern emerged for the shaded drawings, where a significant illusion was found for the differentoriented boxes $[t(20)=3.49, p<.01]$ but not for the sameoriented boxes $[t(20)=0.92]$. This difference in illusion magnitude between the two kinds of shaded drawings was significant [mean difference $=1.76^{\circ}, t(20)=2.56, p<$ $.05]$. The reliability of these results was also confirmed in an experiment run prior to this one, involving 20 different subjects and five of the six stimuli tested here (Figures

Table 1

Mean Alignment Scores and Standard Errors in Experiment 1

\begin{tabular}{lrr}
\hline Stimulus Conditions & $M$ & $S E$ \\
\hline Line Drawings & & \\
$\quad$ Control lines & -1.43 & 0.52 \\
Same orientation & 1.41 & 0.42 \\
Different orientation & 1.56 & 0.62 \\
Shaded Drawings & & \\
Control lines & -1.00 & 0.70 \\
Same orientation & -0.36 & 0.45 \\
Different orientation & 1.40 & 0.75
\end{tabular}

Note - Positive values indicate apparent divergence toward the top of the picture; negative values indicate illusions of convergence.

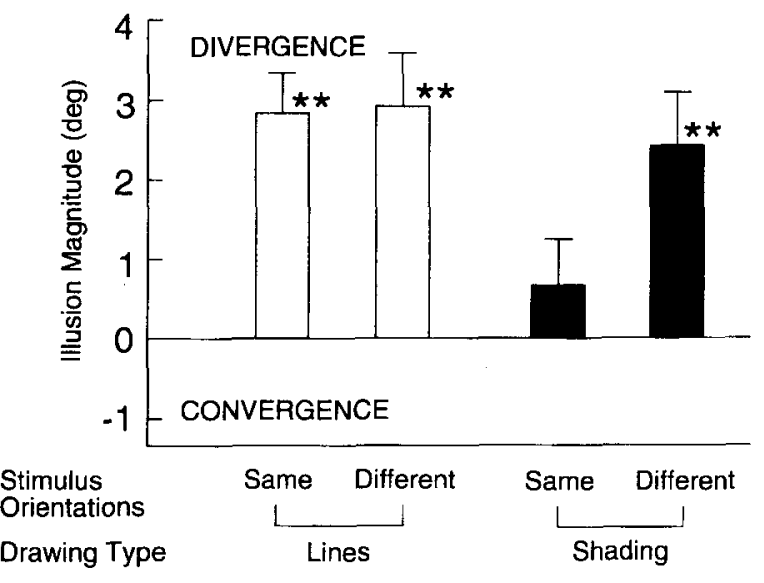

Figure 5. The results of Experiment 1. The box alignment illusion is measured as the difference (in degrees) in alignment settings between various 3-D boxes and corresponding 2-D controls. Double asterisks indicate an illusion of apparent divergence that is significantly different from zero at $p<.01$.

1A, 1B, 1C, 1D, and 1F). The present data pattern was replicated in all essential respects.

The results thus confirm the presence of the box alignment illusion, which manifests itself as the systematic misperception of the orientation of lines that are components of drawings of orthogonally projected objects. However, the pattern of results is also more complex than we had anticipated: (1) settings for the two control stimuli differed significantly from absolute parallel, and (2) a difference in illusion magnitude between same- and differentoriented boxes was apparent in shaded boxes but not in line-drawn ones.

With regard to the first point, our discussion of the illusion has so far been entirely in relative terms (i.e., comparison of the alignments of 3-D boxes vs. 2-D controls). This ignores the possibility that the alignments of the 2-D controls may themselves involve an illusion. That is, there may be two components to the total illusion: one involving an apparent convergence of control lines, and the other involving the apparent divergence of the lines in the boxes. Because it is difficult to distinguish this hypothesis from a simpler one involving a constant error in all alignments, we will reserve discussion of this point until Experiment 2.

Why might the illusion be sensitive to the relative orientations of the two boxes in shaded drawings but not in line drawings? One possibility is that the shaded drawings contribute to a richer 3-D representation and, therefore, to a larger apparent discrepancy in the perceived orientations of the boxes. This is consistent with visual search experiments showing that orientation differences can be detected somewhat more rapidly in shaded boxes than in line-drawn ones (Enns \& Rensink, 1990a, 1990b). Alternatively, the distortion observed for same-oriented boxes may reflect a different illusion. In any case, we were surprised to see a distortion in the same-oriented boxes, and so this effect was also examined more systematically in Experiment 2. 


\section{EXPERIMENT 2 Influence of Interstimulus Distance}

The three line-drawn stimulus pairs (Figures 1A-1C) were tested again in Experiment 2 at three different interstimulus distances (near $=5 \mathrm{~cm}$ between the centers of the two stimuli, middle $=10 \mathrm{~cm}$, and $f a r=15 \mathrm{~cm}$ ). The far distance was thus similar to the distance used for all conditions in Experiment 1. If the misalignments observed in the control lines, same-oriented boxes, and different-oriented boxes reflect different mechanisms, then one might expect distance to have different effects on these stimuli.

\section{Method}

Except for the inclusion of the three distance conditions and a reduction in the number of measurements made by each subject for each stimulus (from five to three), the method was the same as in Experiment 1 . Twenty-five undergraduates ( 16 females, 9 males) from the university subject pool participated in return for partial course credit in psychology. All reported normal or corrected-to-normal acuity, and none had participated in the previous experiment.

\section{Results and Discussion}

The mean alignment scores are shown in Table 2. An ANOVA revealed a significant main effect of stimulus type $\left[F(2,48)=15.30, M S_{\mathrm{e}}=30.71, p<.001\right]$ but not of distance $\left[F(2,48)<1, M S_{\mathrm{e}}=27.40\right]$. However, the stimulus $\times$ distance interaction was significant $[F(4,96)=2.50$, $\left.M S_{\mathrm{e}}=27.36, p<.05\right]$, indicating that the influence of distance varied between the three stimulus types. An examination of the control stimuli revealed a trend toward increased apparent divergence with greater distance [linear $F(1,48)=4.52, p<.04]$. A similar trend was observed for the same-oriented boxes, although the absolute settings began at a higher level $[$ linear $F(1,48)=4.63, p<.04]$. In contrast, the opposite trend was seen for different-oriented boxes, where the illusion of divergence was largest at the nearest distance [linear $F(1,48)=5.10, p<.03$ ] .

Therefore, when the mean illusion magnitude was examined, as shown in Figure 6, only the different-oriented boxes showed a significant illusion of divergence [near, $t(24)=6.14, p<.01$; middle, $t(24)=2.02, p<.05$ (onetail); far, $t(24)<1.0]$. Furthermore, the magnitude of this illusion decreased as the distance between the boxes increased [linear $F(1,48)=6.12, p<.02]$. The small tendency toward an illusion of convergence in the same-oriented boxes was not significantly different from zero, and it did not vary with distance [both $p \mathrm{~s}>.10]$.

Table 2

Mean Alignment Scores and Standard Error in Experiment 2

\begin{tabular}{|c|c|c|c|c|c|c|}
\hline \multirow[b]{3}{*}{ Stimulus Type } & \multicolumn{6}{|c|}{ Interstimulus Distance } \\
\hline & \multicolumn{2}{|c|}{ Near $-5 \mathrm{~cm}$} & \multicolumn{2}{|c|}{ Medium-10 cm } & \multicolumn{2}{|c|}{ Far $-15 \mathrm{~cm}$} \\
\hline & $M$ & $S E$ & $M$ & $S E$ & $M$ & $S E$ \\
\hline Control lines & 0.12 & 0.31 & 0.76 & 0.48 & 1.27 & 0.50 \\
\hline Same orientation & -0.56 & 0.30 & 0.04 & 0.41 & 0.71 & 0.41 \\
\hline Different orientation & 3.91 & 0.42 & 2.76 & 0.53 & 1.81 & 0.68 \\
\hline
\end{tabular}

Note - Positive values indicate apparent divergence toward the top of the picture; negative values indicate illusions of convergence.

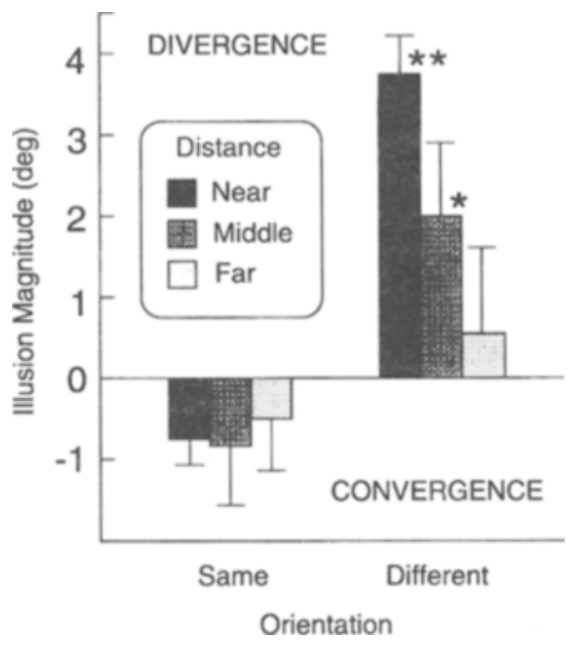

Figure 6. The results of Experiment 2. The box alignment illusion is measured as the difference (in degrees) in alignment settings between various 3-D boxes and 2-D controls. Double asterisks indicate an illusion that is significantly different from zero at $p<.01$.

These data thus confirm the existence of a box alignment illusion for different-oriented boxes. They also confirm that the alignment of the control stimuli is not free from distortion in itself. This was seen in the increase in the distortion of these settings with increasing interstimulus distance. Finally, they show that the illusion observed for different-oriented boxes differs in an important respect from the distortions observed for same-oriented boxes (e.g., in Experiment 1 and here). Whereas the measured distortion in same-oriented boxes increases with interstimulus distance, the distortion for different-oriented boxes is largest at the nearest distance.

\section{THEORETICAL CONSIDERATIONS}

This pattern of results raises interesting questions about the origins of the box alignment illusion. For instance, why should an orientation illusion exist at all in drawings of orthogonally projected objects? Moreover, why should the distortions err on the side of divergence?

It seems likely that the answer will reside, at least to some degree, in the depth cues that give the boxes their appearance of solid 3-D objects. We noted earlier that a number of visual geometric illusions have been attributed to explicit or implicit depth cues (Coren, 1989; Gillam, 1971; Girgus \& Coren, 1973, 1975; Gregory, 1970, Purghé \& Coren, 1992). It is possible to offer some candidate mechanisms for the box alignment illusion that follow a similar line of reasoning.

We will call the first candidate the size constancy hypothesis. It begins with the recognition that there is a form of size constancy illusion in almost all drawings using orthogonal projection. Examples of this can be seen in Figure 7 for two boxes of different dimensions, where the oblique lines delimiting the top face of each box seem to diverge in the picture with increasing apparent distance. 
Notice that the apparent fanning out of the lines seems to stem from the expectation that the parallel lines of an object should converge in a pictorial representation-this expectation is violated in an orthogonal drawing. It therefore seems a reasonable first guess that the apparent "fanning out" of these physically parallel lines may account for the box alignment illusion.

Unfortunately, an explanation based simply on these apparently diverging lines does not adequately account for our data. Consider first the predictions for the control stimuli or the same-oriented boxes. Since the lines in each of the boxes appear to fan out, an apparent illusion of divergence could only be obtained by observers using certain specified pairs of lines for comparison across the two drawings. As can be seen in Figure 8A, if corresponding lines were used (e.g., A1 vs. A2), a parallel alignment would be predicted, or at least a distortion no different from the control lines. However, in half of the remaining comparisons, the lines appear to converge (e.g., C1 vs. A2), whereas, in the other half, they appear to diverge (e.g., A1 vs. C2). Thus, while this hypothesis may predict considerable response variability because of these conflicting biases, it predicts no consistent illusory distortion. Similar predictions for the different-oriented boxes do not fit the data any better. Again, certain corresponding lines appear parallel (e.g., Al with C2), others appear to diverge (e.g., $\mathrm{C} 1$ vs. C2), and still others appear to converge (e.g., Al vs. A2). A satisfactory account of the illusion must therefore go beyond a straightforward extension of size constancy concepts for these stimuli.

We will call a second possible source for the illusion the common-vanishing-point hypothesis. It is based upon the mental representations that observers are believed to form when viewing the depicted objects. For instance, assume that observers consider the orientations of the boxes by first extracting the principle axis of each object (e.g., Biederman, 1987) and then comparing their apparent directions (see the dark lines labeled "A" and "B" in Figure $8 \mathrm{~B}$ ). This is a reasonable assumption, especially since judgments of orientation based upon the local cues (such as the orientation of individual lines, as we discussed above) produce no consistent solution. Next, assume that observers expect the " $B$ " ends of the axes to actually converge, as they would in a true perspective drawing. But, since the boxes are drawn in orthogonal projection, they do not converge. One consequence of these assumptions,
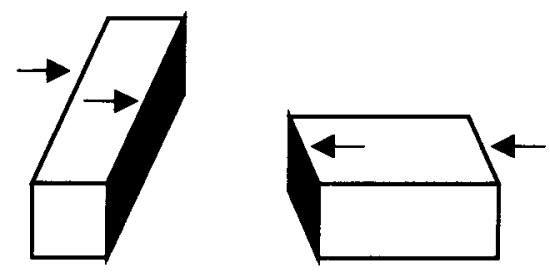

Figure 7. Size constancy mechanisms cause the physically parallel top sides (designated with arrows) of a box drawn in orthogonal projection to appear to diverge with apparent distance (after Coren, Ward, \& Enns, 1994).

A. Size Constancy Hypothesis: parallel edges in orthogonal drawings will appear to fan out when the surfaces they define are receding in depth.
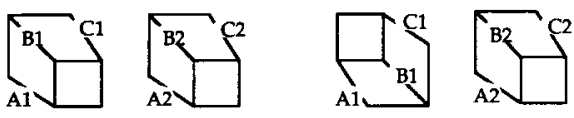

B. Common Vanishing Point Hypothesis: principle axes of boxes are parallel in the picture, but appear to diverge because of an assumed common vanishing point.
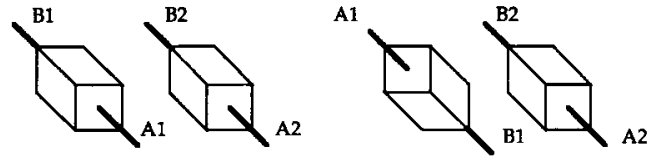

C. Right Angle Hypothesis: different-oriented boxes have parallel oblique edges in the picture plane, but appear to be oriented at right angles in depth.

Stimulus

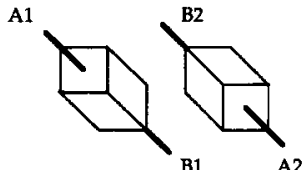

Figure 8. Illustrations corresponding to the three hypotheses considered in understanding the box alignment illusion. All drawings are intended to represent the appearance of the stimuli (in exaggerated form) under various assumptions. See text for details.

then, would be the impression of divergence. A second consequence is that the magnitude of this illusion should increase with interstimulus distance, since to make the principle axes converge to a common vanishing point would create an angle between these axes that increased with interstimulus distance. Both of these predictions were supported by the data for the control and same-oriented stimuli.

Although the common-vanishing-point hypothesis is an adequate explanation of the illusion for these stimuli, it does not work as well for the different-oriented boxes. One problem is that principle axes of these boxes do not appear to lie in the same 3-D plane one box is being viewed from below, the other from above. This can be seen by comparing the labels for the "A-B" axes shown in Figure $8 \mathrm{~B}$, which are in fact reversed in the picture plane with respect to one another. This makes it difficult to know which ends of the axes the common-vanishing-point hypothesis should be applied to. A second problem lies in the data from Experiment 2, where the strongest illusion was observed at the smallest interstimulus distance. As we have said, the common-vanishing-point hypothesis makes the opposite prediction.

A strong clue to what might be going on comes from the reports of observers who were asked questions concerning the depicted relations between the boxes. When observers (both those in the present experiments and others) were asked to position two 3-D hand-held models of the boxes so that they corresponded to the depicted boxes, 
they invariably placed the boxes at or near right angles to one another. We have illustrated this relationship in Figure $8 \mathrm{C}$ and have called it the right-angle hypothesis. It is important to note that if observers are assuming this to be the depicted relation between the boxes, then the alignment task is indeed presenting them with a conflict. Retinally, the oblique lines in each box are parallel to one another; however, in the mental representation of the situation, the boxes are much closer to one another at one end than the other. If observers are being influenced by such mental representations, it should result in an illusion of divergence. Furthermore, decreasing interstimulus distance would serve to heighten the disparity between the mental representation of the boxes and the lines in the drawing, just as we found in Experiment 2.

In summary, we propose that subjects make several implicit assumptions about the 3-D objects rendered in orthogonal projection. Among these is that lines from objects with similar 3-D orientations are expected to converge to a common vanishing point in the picture. Thus, failure of these depicted lines to actually converge will be seen as apparent divergence. A second assumption is that objects drawn to represent different 3-D orientations will appear to be oriented toward different implicit vanishing points. A direct consequence of using physically parallel edges then, to represent objects in different 3-D orientations, is that these parallel edges will appear to diverge.

\section{EXPERIMENT 3 Effects of Pictorial Depth in the Background}

To test the generality of our account, we examined the influence of strong cues to pictorial depth in the immediate surround of the stimuli being aligned.

How might a background with strong pictorial depth cues influence the alignment settings? First, it might have a direct influence on the setting of the control stimuli. Just as the 3-D context in Figure 3 seems to have an influence on the appearance of an ambiguous 2-D component (the individual lines on the side of the box), so might a pictorial background with strong cues to depth have an influence on the setting of the control lines. In particular, placing an explicit vanishing point in the immediate context of the lines might serve to enhance an illusion of divergence, since instead of appearing only slightly divergent with respect to an implicit vanishing point in the far distance, the lines might now appear to be highly divergent with respect to an explicit vanishing point that is relatively near.

Another possible influence could involve the relation between the relative orientations of the depicted objects and the number of vanishing points in the background. For example, two boxes with the same 3-D orientations should be evaluated with respect to the same explicit vanishing point, and so the degree of distortion in these displays should be insensitive to the number of depicted vanishing points. In contrast, the interpretation of two boxes with different orientations could be influenced by whether there is one or two explicit vanishing points. Two vanishing points that are quite separate from one another in the de- picted scene would permit each box to be evaluated separately; in contrast, one vanishing point alone might produce a conflict between the reference orientation of the standard box and those of the background surfaces.

The four different pictorial backgrounds tested are shown in Figure 9. Since these backgrounds are all highly realistic renderings, they differ in a large number of uncontrolled ways. However, we chose this set because of the obvious differences between them in the number of vanishing points depicted. Figures 9A and 9B contain two (one toward the right and one toward the left of the picture), whereas Figures $9 \mathrm{C}-9 \mathrm{D}$ contain only one (each in the center). A fifth background tested contained no explicit vanishing points and so served as a control (blank background).

\section{Method}

Four different groups of 20 observers were tested. One group adjusted pairs of control lines ( 9 females, 11 males), another adjusted line-drawn same-oriented boxes ( 10 females, 10 males), another adjusted line-drawn different-oriented boxes (11 females, 9 males), and a fourth group adjusted shaded boxes in different orientations ( 9 females, 11 males). Five settings were made for each background condition in a random order. In all other respects, the method was identical to the previous experiments.

\section{Results and Discussion}

The mean alignment scores are shown in Table 3. The most striking feature of the data was a reversal in the direction of the illusion. There was now an illusion of divergence in the control lines (overall mean $=1.30$ ), whereas the tendency for the box stimuli was toward an illusion of convergence (overall mean $=-0.63$ ). Furthermore, the magnitude of these distortions was influenced by the background. Two vanishing points (Figures 9A and 9B) produced more illusory divergence in the control lines than did one vanishing point (Figures 9C and 9D) [mean difference $=1.18, t(152)=3.48, p<.01]$. An opposite pattern was observed for the different-oriented boxes. These stimuli produced a stronger illusion of convergence in the presence of two vanishing points than in the presence of only one [mean difference $=0.77, t(152)=2.28, p<.05]$. An ANOVA showed that the different backgrounds had a highly significant effect overall $\left[F(2,152)=16.91, M S_{\mathrm{e}}=\right.$ $2.29, p<.001]$, that the effect of stimulus type was significant $\left[F(3,76)=2.31, M S_{\mathrm{e}}=14.56, p<.08\right]$, and that their interaction was significant $\left[F(6,152)=4.63, M S_{\mathrm{e}}=\right.$ $2.29, p<.001]$.

The overall magnitude of the box alignment illusion was computed for each of the background conditions in the same way as for previous experiments (i.e., the mean setting for the control lines was subtracted from that of the boxes, separately for each background condition). These results are shown in Figure 10.

For the same-oriented boxes, there was a significant illusion for both the one- and the two-vanishing-point conditions, but not for the blank background. Planned comparisons indicated no difference between the one- and the two-vanishing-point conditions $[t(19)=0.73]$. However, there was a significant reduction in the illusion when all depicted depth cues were removed $[t(19)=4.72, p<.001]$. 

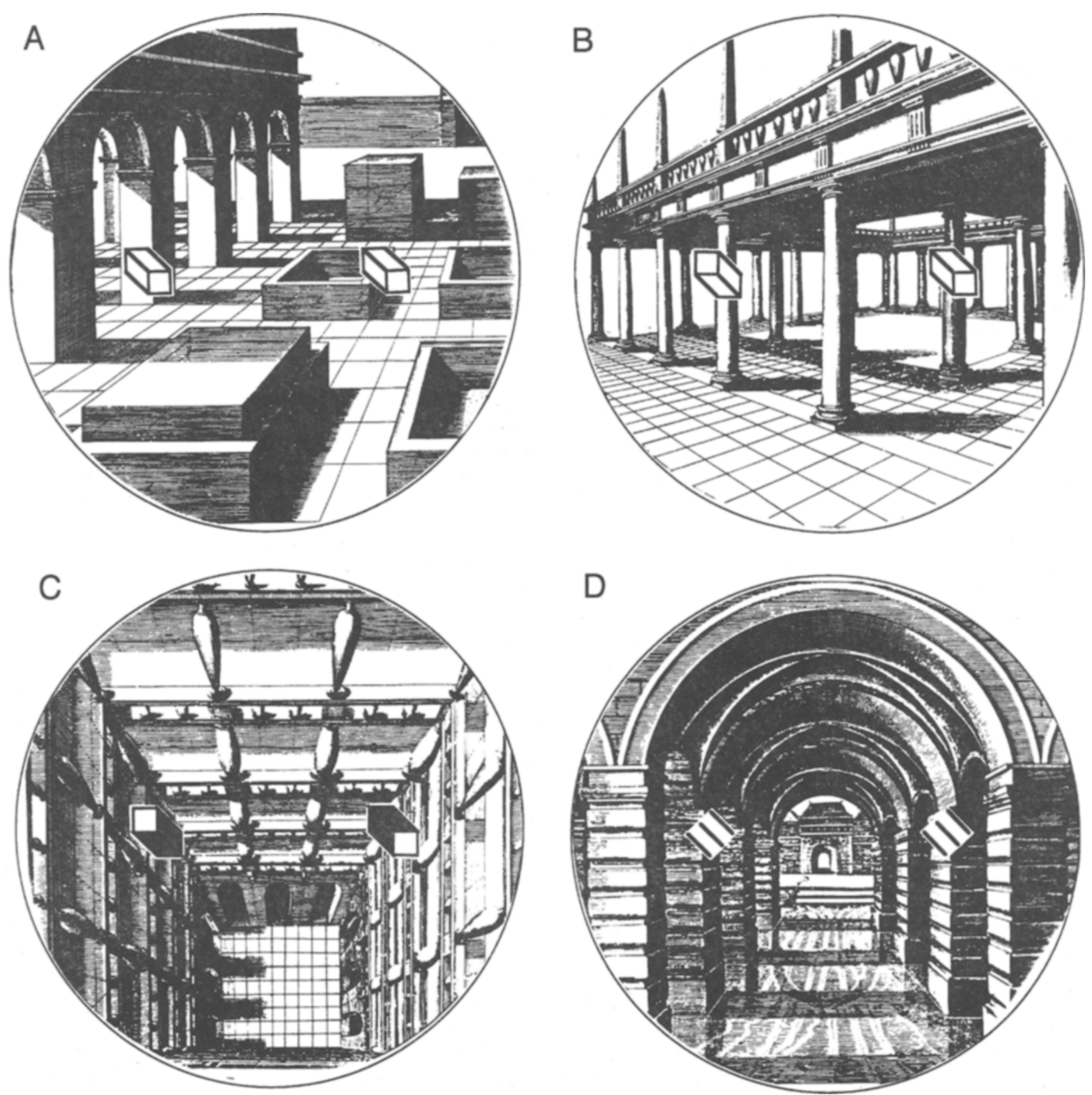

Figure 9. The four pictorial backgrounds used in Experiment 3. A and B contain two vanishing points; C and D contain only one. The pairs of stimuli shown against each background are examples of the stimuli that were aligned in the experiment.

Thus, it seems that as long as vanishing points are depicted on the background, an illusion of convergence is obtained for same-oriented boxes, with the number of vanishing points not affecting illusion magnitude.

The different-oriented boxes were sensitive to the number of vanishing points in both their line-drawn and their shaded forms. For line-drawn boxes, the only significant illusion of convergence was for the two vanishing points $[t(19)=4.76$, $p<.001]$. Planned comparisons show that the illusion for two vanishing points was significantly greater than that for only one $[t(19)=4.20, p<.001]$. There was, however, no difference between the one-vanishing-point background and the blank background $[t(19)=1.21]$. A similar picture emerged for the shaded boxes. Although both the two- and the onevanishing-point conditions yielded a significant illusion $[t(19)=3.53, p<.01 ; t(19)=2.21, p<.05]$, the twovanishing-point illusion was also significantly greater than that one-vanishing-point illusion $[t(19)=3.27, p<.01]$, and there was no significant difference between the illusion for one vanishing point and that for none $[t(19)=0.93]$.

Thus, a first major finding of Experiment 3 was that settings of oblique control lines were strongly influenced by the pictorial backgrounds, with two vanishing points producing a larger illusion of divergence than only one. Additional testing will be required to determine whether the determining factors are the number of depicted vanishing 
Table 3

Mean Alignment Scores and Standard Errors in Experiment 3

\begin{tabular}{|c|c|c|c|c|c|c|c|c|}
\hline \multirow[b]{2}{*}{ Background } & \multicolumn{2}{|c|}{ Control Lines } & \multicolumn{2}{|c|}{ Same Lines } & \multicolumn{2}{|c|}{ Different Lines } & \multicolumn{2}{|c|}{ Different Shading } \\
\hline & $M$ & $\overline{S E}$ & $M$ & $S E$ & $M$ & $S E$ & $M$ & $S E$ \\
\hline \multicolumn{9}{|c|}{ Two Vanishing Points } \\
\hline Background A & 1.96 & 0.62 & -1.18 & 0.44 & -0.46 & 0.76 & -1.16 & 1.16 \\
\hline Background B & 1.82 & 0.59 & -0.27 & 0.40 & -1.14 & 1.19 & -0.99 & 1.31 \\
\hline \multicolumn{9}{|c|}{ One Vanishing Point } \\
\hline Background C & 0.91 & 0.54 & 0.19 & 0.74 & 0.15 & 0.81 & -0.61 & 1.00 \\
\hline Background D & 0.52 & 0.76 & -1.77 & 0.72 & 0.28 & 0.67 & -0.48 & 1.02 \\
\hline \multicolumn{9}{|l|}{ Blank Background } \\
\hline Background $\mathrm{E}$ & 1.53 & 0.57 & 1.77 & 0.55 & 0.40 & 0.70 & 0.76 & 1.05 \\
\hline
\end{tabular}

Note-Positive values indicate apparent divergence toward the top of the picture; negative values indicate illusions of convergence.

points, the location(s) of the vanishing point(s), or some other factors as yet unaccounted for.

A second finding was the reversal of the box alignment illusion (at least relative to the controls) when the stimuli were presented against a background with pictorial depth. We had speculated that the depicted depth might affect the magnitude of the box alignment illusion because the orientation of the boxes would be evaluated with respect to the location of one or more explicit vanishing points. Such points, which could now serve as a reference for the ambiguous orientation of the orthogonally drawn boxes, may have influenced observers to see the boxes as part of the larger picture. As such, the orientation of the boxes would be judged with reference to the perspective lines in the background. Since these lines do converge to a common vanishing point, the observer may have also assumed, with some justification, that the principle axes of the boxes were converging. The distorted alignments may therefore have reflected an attempt to compensate for this apparent convergence.

The third finding concerned the effect of vanishing-point number on the boxes. Our account suggested that this variable would not affect the same-oriented boxes, since only one vanishing point is of relevance for two objects with the

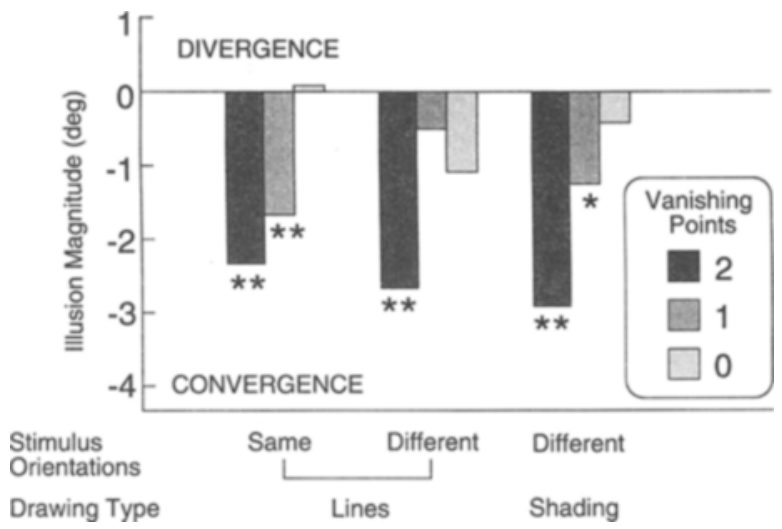

Figure 10. The results of Experiment 3. The box alignment illusion is measured as the difference (in degrees) in alignment settings between 3-D boxes and 2-D controls. Double asterisks indicate an illusion that is significantly different from zero at $p<.01$; single asterisks indicate an illusion that is significantly different from zero at $p<.05$. same orientation in 3-D space. However, more than one vanishing point might influence the appearance of the differentoriented boxes as follows: With only one vanishing point, these boxes could easily be seen as standing at roughly right angles to one another. Indeed, if anything, only one explicit vanishing point should reinforce such an interpretation, since the shorter axis of one box could be interpreted as pointing in the same direction as the longer axis of the other box (see Figure $8 \mathrm{C}$ ). With two vanishing points, the orientation of each box could be interpreted with respect to a separate point, thereby undermining the interpretation that the boxes stand at right angles to one another. Interestingly, the vanishing points on the right side of both Figure $9 \mathrm{~A}$ and Figure $9 \mathrm{~B}$ are higher in the picture than the extension of the principle axis of the rotatable box on the left side. This may have served to exert an influence on the stronger convergence illusion observed with these two backgrounds.

Finally, we note a failure to replicate one aspect of the results of Experiments 1 and 2 in these data. For the blank background there is no longer a significant illusion of divergence. In fact, the direction of the illusion in the two different-orientation conditions, although only significant in one case, is actually in the opposite direction (convergence). However, obtaining this finding in the present context may actually support some aspects of our reasoning. Since the blank and pictorial backgrounds were presented intermixed, it seems reasonable that the schema ordinarily used in the blank-background condition may have been influenced by the pictorial context. Similar "carryover" or set effects have been shown to influence visual illusions of various classes by attenuating or even reversing the distortions usually obtained (e.g., Coren, 1984, 1986; Coren \& Porac, 1983a; Coren, Porac, \& Theodor, 1986). If this is the case, then using the same blank background and measuring apparatus as in Experiment 3, in the absence of other pictorial stimuli, ought to restore the divergence illusion that we found earlier. To verify that this was indeed the case, we conducted Experiment 4.

\section{EXPERIMENT 4 Control for Perceptual Set}

The 2-D control lines and the two different-oriented 3-D box conditions were tested in Experiment 4, in each 
case presented only against the blank background. Thus, the observers in this experiment were never shown the pictorial backgrounds that might bias their presumptions as to the locus of the implied vanishing points.

\section{Method}

Control lines, different-oriented line-drawn boxes, and differentoriented shaded boxes were used as stimuli. Otherwise, the method was the same as Experiment 3, with five settings per stimulus in a random order. Twenty undergraduates ( 9 females, 11 males) from the university subject pool participated in return for partial course credit in psychology. All reported normal or corrected-to-normal acuity, all were naive to the purpose of the experiment, and none had participated in any of the previous experiments.

\section{Results and Discussion}

An ANOVA revealed a significant main effect of drawing type $\left[F(2,38)=3.22, M S_{\mathrm{e}}=11.29, p<.05\right]$, consistent with a diverging box alignment illusion. These data were treated in the same way as in the previous experiments, with the box settings being compared with the control line settings. However, unlike the previous experiments, there was now a significant effect of trials on the illusion scores $\left[F(4,76)=2.44, M S_{\mathrm{e}}=20.06, p<.05\right]$, and so the data for the five trials are shown separately in Table 4 and Figure 11. Only the individual comparisons in the first two trials were significant for each drawing type $(p<.01)$. The values in the two drawing styles did not differ reliably for any of the trials in which there was a significant illusion.

These results confirm that the box alignment illusion of apparent divergence is in fact present when the boxes are shown against a blank background with no inducing context of depicted depth. Together with the data in the previous experiments, they strongly suggest that the trend toward a reversal of the illusion in the blank background in Experiment 3 was a carryover or set effect that influenced the 3-D processing mechanisms brought to bear on the task.

The significant decrement of the illusion observed here is interesting because it is reminiscent of the typically reported decrement effect that occurs when one freely inspects an illusion figure over time (e.g., Coren, 1991; Coren \& Girgus, 1974; Coren, Girgus, \& Schiano, 1986; Porac $\&$ Coren, 1985). The fact that it was not significant in the previous experiments is probably because they each employed a larger number of figures that were randomly intermixed. If this interpretation is correct, it provides additional support for the multicausal nature of the box alignment illusion.

Table 4

Mean Alignment Scores and Standard Errors in Experiment 4

\begin{tabular}{|c|c|c|c|c|c|c|}
\hline \multirow[b]{2}{*}{ Trial } & \multicolumn{2}{|c|}{ Control } & \multicolumn{2}{|c|}{ Line Box } & \multicolumn{2}{|c|}{ Shaded Box } \\
\hline & $M$ & $S E$ & $M$ & $S E$ & $M$ & $S E$ \\
\hline 1 & -0.55 & 0.44 & 1.50 & 0.94 & 2.30 & 0.98 \\
\hline 2 & 0.20 & 0.37 & 2.20 & 1.12 & 2.05 & 0.94 \\
\hline 3 & 0.05 & 0.48 & 0.70 & 0.65 & -0.50 & 0.67 \\
\hline 4 & 0.10 & 0.51 & 0.85 & 0.96 & 0.65 & 0.94 \\
\hline 5 & 0.25 & 0.35 & 0.50 & 0.55 & 0.10 & 0.66 \\
\hline
\end{tabular}

Note-Positive values indicate apparent divergence toward the top of the picture; negative values indicate illusions of convergence.

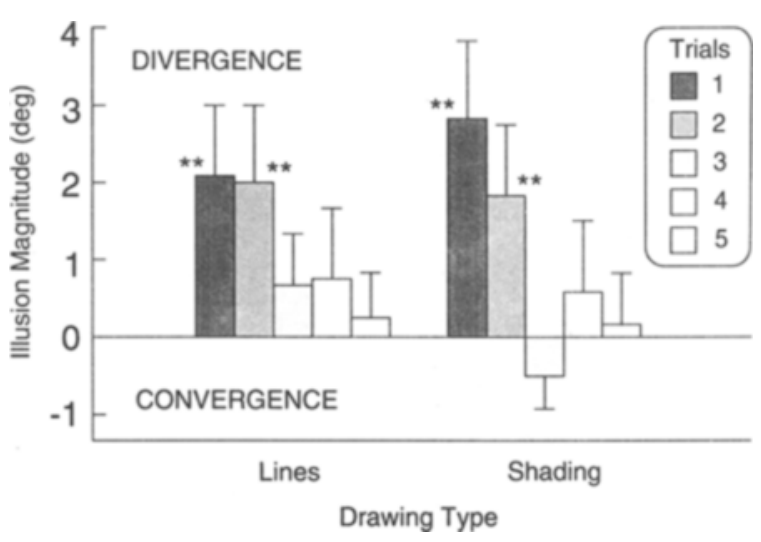

Figure 11. The results of Experiment 4. The box alignment illusion is measured as the difference (in degrees) in alignment settings between 3-D boxes and 2-D controls. Double asterisks indicate an illusion that is significantly different from zero at $p<.01$.

\section{GENERAL DISCUSSION}

The results of these four experiments clearly demonstrate the existence of a new illusion. Stated as simply as possible, the box alignment illusion appears as a systematic distortion in the alignment of 3-D objects drawn with obliquely oriented parallel lines. Relative to flat-appearing control lines and to 3-D boxes with the same orientation, the edges of differently oriented 3-D boxes appear to diverge toward the top of the picture.

It is important to note here that there does not appear to be any control condition that is itself immune from distortions in the alignment settings. As shown in Experiments 2 and 3 , even the settings made for triplets of oblique parallel lines are influenced by variables such as interstimulus distance and the immediate context of pictorial depth cues. Settings made for same-oriented boxes are also not free from distortion, although they do seem to be governed by different variables. Therefore, as with other illusions of orientation (Hotopf et al., 1983; Wenderoth \& Johnson, 1985), caution should be used in interpreting the raw values from the alignment task. However, the relative differences that are observed certainly appear to be amenable to systematic study.

The box alignment illusion clearly behaves in a manner that suggests the involvement of mechanisms used in pictorial depth perception. In particular, we propose that observers' settings are influenced by implicit assumptions that are normally very beneficial in extracting depth from pictures. Such assumptions include the following: (1) The rough orientation of an object drawn in orthogonal projection is given by its principle axis. (2) The extended principle axes of two objects with the same depicted orientation should eventually converge on the horizon. (3) The extended principle axes of two objects with different depicted orientations should intersect one another in the picture plane. Violations of these assumptions in a drawing can lead directly to the kinds of distortions we observed. For example, there is a distortion inherent in every orthog- 
onal drawing, since the parallel edges of an object fail to converge in the picture as expected. Second, even if this distortion is overcome in the interpretation of each object in the drawing, the expectation that the principle axes of objects of similar 3-D orientation will converge in the picture plane will be violated, leading to another distortion (apparent divergence of two same-oriented boxes). Finally, if physically parallel edges in the picture are used to represent edges with different 3-D orientations in the scene, then the boxes will appear to be closer to one another at one end than at the other.

If this analysis is correct, it means that the box alignment illusion is similar to other illusions in which a 3-D interpretation influences what is ostensibly a 2-D judgment (see Coren \& Girgus, 1978). What makes the box alignment illusion unique is that it appears to be sensitive to more than one aspect of pictorial perception. This suggests that explanations that try to unify all aspects of the distortion on the basis of a single mechanism or cue are most likely doomed to failure. On the other hand, because of its multicausal nature, the box alignment illusion may prove useful in the study of a large range of issues.

The box alignment illusion therefore serves as a new demonstration of the strong bias of human observers to interpret line drawings as representations of objects and surfaces in 3-D space. Its existence is even more striking when one considers how poorly orthogonal drawings actually represent the $3-\mathrm{D}$ world visible to the eye. The depicted boxes do not contain perspective information (i.e., lines should converge toward a vanishing point), the front face of each box is drawn in the frontoparallel plane (i.e., this is inconsistent with seeing the side and top faces at the same time), and the surfaces of the box either are not presented (i.e., in the line drawings) or are shown with uniform brightness values in the shaded drawings (i.e., shading is graded in naturalistic scenes). Nonetheless, observers involuntarily interpret the 3-D shape and orientation of these boxes in a systematic way.

We readily admit that we have only scratched the surface of this illusion. Future work will need to examine such questions as: To what extent is the illusion dependent on drawings rendered in orthogonal projection (i.e., parallel edges in an object are parallel lines in the drawing)? How does variation in the apparent height in the picture plane or the specific locus of vanishing points affect the magnitude of the distortion? Are only oblique lines subject to this illusion, or does the illusion occur generally for lines of all orientations? Whatever the outcome, we believe this illusion will prove useful in helping us understand visual perception, both of pictorial renderings and of scenes more generally.

\section{REFERENCES}

Biederman, I. (1987). Recognition-by-components: A theory of human image understanding. Psychological Review, 94, 115-147.

BORRESEN, R. C. (1990). Illusion reversal rate as a function of subjective depth. Journal of General Psychology, 117, 39-46.

Bruno, N., \& Cutting, J. E. (1988). Minimodularity and the percep- tion of layout. Journal of Experimental Psychology: General, 117, $161-170$.

Butler, D. L., \& KRING, A. M. (1987). Integration of features in depictions as a function of size. Perception \& Psychophysics, 41, 159-164.

CARPENTER, R. H. S., \& BlaKEMORE, C. (1973). Interactions between orientations in human vision. Experimental Brain Research, 18, 287-303.

COREN, S. (1972). Subjective contour and apparent depth. Psychological Review, 79, 359-367.

COREN, S. (1984). Set. In R. J. Corsini (Ed.), The encyclopedia of psychology (Vol. 3, pp. 296-298). New York: Wiley.

COREN, S. (1986). An efferent component in the visual perception of direction and extent. Psychological Review, 93, 391-410.

Coren, S. (1989). The many moon illusions: An integration through analysis. In M. Hershenson (Ed.), The moon illusion (pp. 351-370). Hillsdale, NJ: Erlbaum

COREN, S. (1990). Perceptual constancies. In M. W. Eysenck (Ed.), The Blackwell dictionary of cognitive psychology (pp. 255-257). Oxford, U.K.: Basil Blackwell.

Corian, S. (1991). Visual illusions. In R. Dulbecco (Ed.), Encyclopedia of human biology (Vol. 7, pp. 829-833). Orlando, FL: Academic Press.

COREN. S. (1992). The moon illusion: A different view through the legs. Perceptuai \& Motor Skills, 75, 827-831.

COREN, S., \& AKS, D. J. (1990). Moon illusion in pictures: A multimechanism approach. Journal of Experimental Psychology: Human Perception \& Performance, 16, 365-380.

COREN, S., \& Girgus, J. S. (1974). Transfer of illusion decrement as a function of perceived similarity. Journal of Experimental Psychology, 102, 881-887.

COREN, S., \& Girgus, J. S. (1975). A size illusion based upon a minimal interposition cue. Perception, 4, 251-254.

Coren, S., \& Girgus, J. S. (1977). Illusions and Constancies. In W. Epstein (Ed.), Stability and constancy in visual perception: Mechanisms and processes (pp. 255-283). New York: Wiley.

Coren, S., \& Girgus, J. S. (1978). Seeing is deceiving: The psychology of visual illusions. Hillsdale, $\mathrm{NJ}$; Erlbaum

Coren, S., Girgus, J. S., \& Schiano, D. (1986). Is adaptation of orientation-specific cortical cells a possible explanation illusion decrement? Bulletin of the Psychonomic Society, 24, 207-210.

COREN, S., \& HoY, V.S. (1986). An orientation illusion analog to the rod and frame: Relational effects in the magnitude of distortion. Perception \& Psvchophysics, 39, 159-163.

COREN, S., \& PORAC, C. (1983a). The creation and reversal of the MüllerLyer Illusion through attentional manipulation. Perception, 12, 49-54.

Coren, S., \& Porac, C. (1983b). Subjective contours and apparent depth: A direct test. Perception \& Psychophysics, 33, 197-200.

Coren, S., Porac, C., \& Theodor, L. H. (1986). The effects of perceptual set on the shape and apparent depth of subjective contours. Perception \& Psychophysics, 39, 327-333.

Coren, S., WART, L. M., \& ENNS, J. T. (1994). Sensation and perception (4th ed.). Fort Worth, TX: Harcourt Brace.

Deregowski, J. B. (1989). Real space and represented space: Crosscultural perspectives. Behavioral \& Brain Sciences, 12, 51-119.

EnNS, J. T., \& Rensink, R. A. (1990a, February 9). Influence of scenebased properties on visual search. Science, 247, 721-723.

ENNS, J. T., \& RENSINK, R. A. (1990b). Sensitivity to three-dimensional orientation in visual search. Psychological Science, 1, 323-326.

Enns, J. T., \& Rensink, R. A. (1991). Preattentive recovery of threedimensional orientation from line drawings. Psychological Review, 98, 335-351.

GiBSON, J. J. (1979). The ecological approach to visual perception. Boston: Houghton-Mifflin.

GILLAM, B. (1971). A depth processing theory of the Poggendorff illusion. Perception \& Psychophvsics, 10, 211-216.

Gillam, B. (1980, January). Geometrical illusions. Scientific American, 242, 102-111

Girgus, J. S., \& COREN, S. (1973). The stability of forms under conditions of illusory distortion. Perceptual \& Motor Skills, 37, 715-719.

Girgus, J. S., \& COREN, S. (1975). Depth cues and constancy scaling in the horizontal-vertical illusion: The bisection error. Canadian Journal of Psychology, 29, 59-65.

Gregory, R. L. (1970). The intelligent ele. New York: McGraw-Hill 
Gregory, R. L., \& Harris, J. B. (1974). Illusory contours and stereo depth. Perception \& Psychophysics, 15, 411-416.

Hotopf, W. H. N., Hibberd, M. C., \& Brown, S. A. (1983). Position in the visual field and spatial expansion. Perception, 12, 469-476.

HowARD, I. P. (1982). Human visual orientation. Chichester, U.K: Wiley.

KaufMan, L., \& Rock, I. (1989). The moon illusion thirty years later. In M. Hershenson (Ed.), The moon illusion (pp. 193-234). Hillsdale, NJ: Erlbaum.

KenNedy, J. M. (1974). A psychology of picture-perception: Information and images. San Francisco: Jossey-Bass.

KLEFFNER, D., \& RamaChandRan, V. S. (1992). On the perception of shape from shading. Perception \& Psychophysics, 52, 18-36.

Lawson, R. B., Cowan, E., Gibbs, T. D., \& Whitmore, C. T. (1974). Stereoscopic enhancement and a ratio of subjective contours. Journal of Experimental Psychology, 103, 1142-1146.

Niall, K. K., \& Macnamara, J. (1990). Projective invariance and picture perception. Perception, 19, 637-660.

Parks, T. E., \& Hui, L. (1989). Pictorial depth and the Poggendorff illusion. Perception \& Psychophysics, 46, 465-468.

Perkins, D. N. (1972). Visual discrimination between rectangular and nonrectangular parallelopipeds. Perception \& Psychophysics, 12, 396-400.

Porac, C., \& CoREN, S. (1985). Transfer of illusion decrement: The effects of global versus local figural variations. Perception \& Psychophysics, 37, 515-522.

PurghÉ, F., \& CoRen, S. (1992). Amodal completion, depth stratification, and illusory figures: A test of Kanizsa's explanation. Perception, 21, 325-335.

RAMACHANDRAN, V. S. (1988, August). Perceiving shape from shading. Scientific American, 259, 76-83.
REED, C. F. (1984). Terrestrial passage theory of the moon illusion. Journal of Experimental Psychology: General, 113, 489-500.

Rochlin, A. M. (1955). The effect of tilt on the perception of parallelness. American Journal of Psychology, 68, 223-236.

voN COLLANI, G. (1985). The horizontal-vertical illusion in photographs of concrete scenes with and without depth information. Perceptual \& Motor Skills, 61, 523-531.

VuRPILlot, E. (1963), L'organization perceptive: Son role dans l'evolution des illusions optico-geometriques. Etudes de psychologie et de philosophie XVI [The organization of perception: Its role in the development of visual-geometric illusions. Studies in psychology and philosophy.]. Paris: Librarie Philosophique J. Vrin.

WADE, N. (1990). Visual allusions: Pictures of perception. Hillsdale, NJ: Erlbaum.

Ward, L. M., Porac, C., Coren, S., \& Girgus, J. S. (1977). The case for misapplied constancy scaling: Depth associations elicited by illusion configurations. American Journal of Psychology, 90, 609-620.

WARREN, R. M., \& BASHFord, J. A. (1977). Müller-Lyer illusions: Their origin in processes facilitating object recognition. Perception, $\mathbf{6}$, 615-626.

WeissteIn, N., \& Harris, C. S. (1974, November). Visual detection of line segments: An object superiority effect. Science, 186, 752-755.

WENDEROTH, P., \& JOHNSON, M. (1985). What is the appropriate control for the tilt illusion? Perception, 14, 275-283.

(Manuscript received March 10, 1994; revision accepted for publication April 1, 1995.) 